\title{
Imaging Adaptive Immune Response in Single Cells using TIRF Microscopy
}

\author{
J.S. Aaron*, A. Carroll-Portillo**, J.R. Pfeiffer**, B.S. Wilson** \& J.A Timlin* \\ * Bioenergy and Defense Technology Dept., Sandia National Laboratories, Albuquerque, NM 87185 \\ ** Dept. Pathology, University of New Mexico Health Sciences Center, Albuquerque, NM 87131
}

The complex trafficking of membrane-associated proteins plays an important role in antigen recognition and immune response. Vital to a comprehensive understanding of immune responses is the early spatial-temporal behavior of proteins at the membrane. We chose to study the dynamics of the high-affinity IgE receptor (FceRI) in mast cells [1,2] following addition of its stimulatory ligand dinitrophenol (DNP). This system represents a well-studied, yet not fully understood molecular mediator of acquired immune system stimulation. Receptor dynamics and secretory vesicle trafficking were followed simultaneously in living cells with total internal reflection fluorescence microscopy (TIRF-M). We chose to visualize secretory lysosomes using the apoptosis-causing Fas ligand (FasL) as it has been shown to be targeted to secretory lysosomes within mast cells. Following antigen stimulation and receptor crosslinking the intracellular signaling cascade is initiated. This cascade results in secretory vesicles fuse to the cell membrane and release their contents into the extracellular space [2]. While the identity of these and other molecular players are known, as well as their relative roles in immune response signaling cascades, relatively little work has been done in studying immune response in the stochastic regime. Optical microscopy provides an opportunity to evaluate single cell behavior, thus providing a more complete, nuanced view of this system.

TIRF microscopy, in particular, lends itself well to studies of membrane-associated proteins, as it confines the excitation to the cell-antigen interface [3]. We synthesized supported lipid bilayers containing up to $251 \%(\mathrm{~mol} / \mathrm{mol})$ dinitrophenol (DNP). RBL mast cells previously transfected with FasL:GFP fusion protein, and incubated with Dy-IgE fluorescent conjugates were deposited on the DNP-lipid bilayers. Due to the large Stokes' shift of the Dy dye, both fluorophors were excited by a single $488 \mathrm{~nm}$ laser and monitored simultaneously on a single CCD detector.

Figures 1 and 2 highlight our results. Figure 1A contains TIRF images of IgE receptor clusters (shown in red) and FasL expression within the secretory vesicles (green). A large-scale reorganization towards the central portion of the interface is observed within 5-10 minutes of cell contact with the stimulatory substrate. We hypothesize that this is made possible by the 2D fluidity of the DNP-containing lipid bilayer. Similar large-scale IgE reorganization is not observed when antigen mobility is restricted via glutaraldehyde tethering of DNP to a glass surface (B, left and middle), or if stimulated cells are in contact with an untreated glass surface (B, right). Figure 2, left indicates DNP-mediated FasL degranulation (shown in green) in RBL cells at early time-points upon contact with the DNP-lipid bilayer. Images were acquired at $10 \mathrm{fps}$. Upon fusing with the cell membrane, FasL-containing secretory vesicles become apparent as "bursts" in the signal intensity, as shown in Figure 2, right. Further image analysis will be presented in an effort to elucidate the dynamics of these molecular mediators of immune response. This work demonstrates the utility of TIRF microscopy for imaging localized molecular behavior in response to immune challenge with both high spatial and temporal resolution, and provides insight into the early, middle, and late phases of immune responses. Visualization of these processes at the single cell level is critical to provide 
fundamental understanding of both the innate and acquired immune response and thus improved treatment of disease.

\section{References:}

[1] Kinet, J.-P., Ann. Rev. Iimmunol., 1999. 17(1): p. 931-972.

[2] Smith, et al., Traffic, 2003. 4:p. 302-312

[3] Axelrod, D., Traffic, 2001. 2(11): p. 764-774.

[4] Sandia is a multiprogram laboratory operated by Sandia Corporation, a Lockheed Martin Company, for the United States Department of Energy's National Nuclear Security Administration under contract DE-AC04-94AL85000.

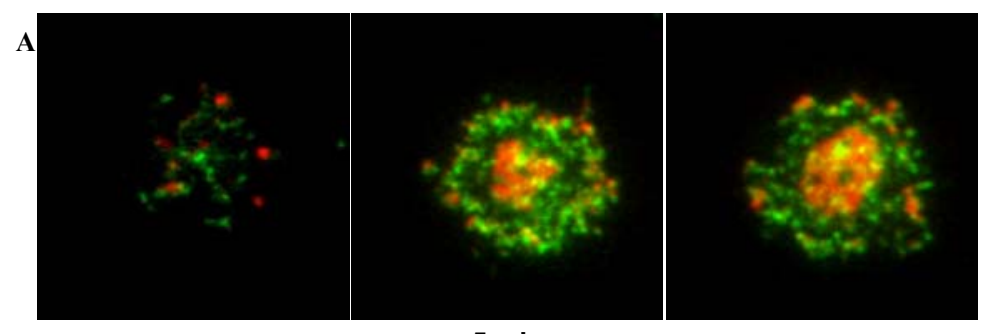

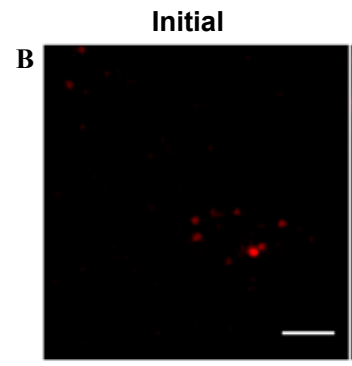

Initial
5 min.

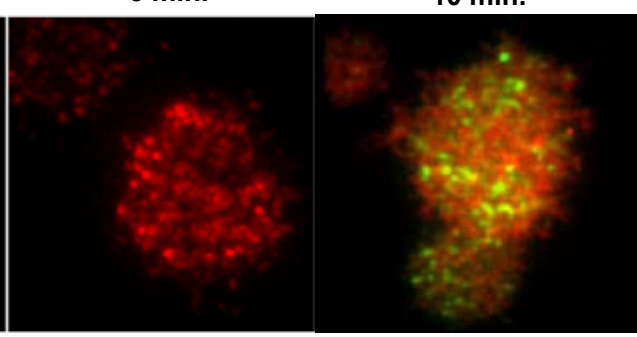

$5 \mathrm{~min}$.

Glass

FIG 1. TIRF microscopy images of RBL mast cells transfected with GFP:FasL (green) and labeled with Dy-conjugated IgE molecules (red). In (A), cells were allowed to adhere to a POPC lipid bilayer containing 25 mol-\% DNP antigen. In (B, left and middle), RBL cells are shown adhered to immobilized DNP-coated substrates and on untreated glass (B, right). Scale bar is ca. $10 \mu \mathrm{m}$.
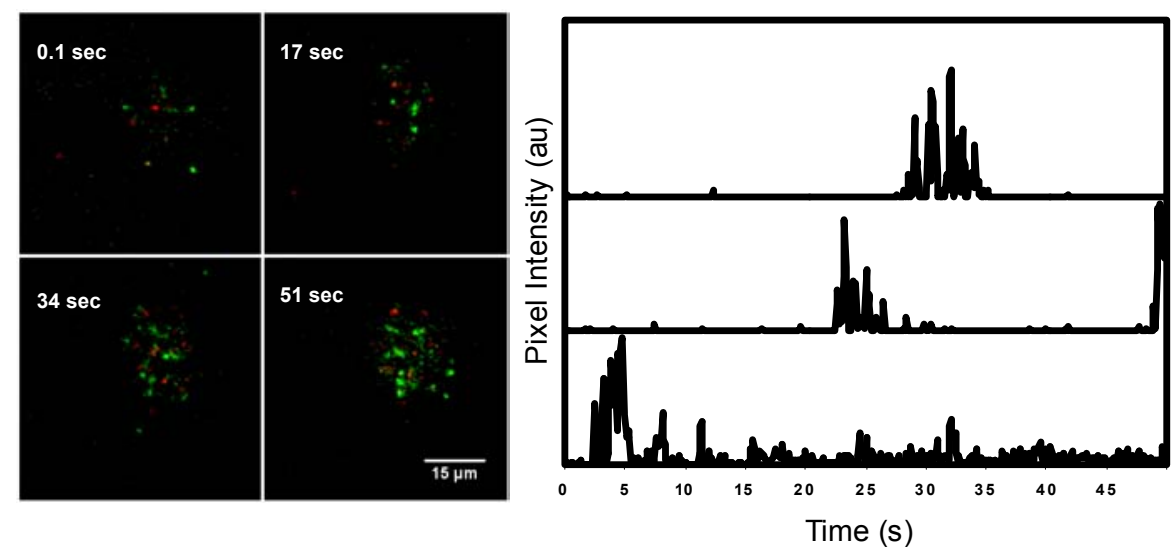

FIG 2. Single cell degranulation assay. At left, TIRF images reveal degranulation events (shown in green) in RBL cells transfected with FasL:GFP. Initial behavior is captured at $>10 \mathrm{fps}$, as cells come in contact with DNP-containing POPC lipid bilayers. At right, signal intensity profiles are plotted at three individual pixel locations over time. Note the signal "bursts" as secretory lysosomes fuse with the cell membrane, releasing their contents into the cell-bilayer interface. 